and accelerators on Portland cement, reported by Dr. L. Forsén, has shown that their effects are dependent on the rate at which they cause precipitation of alumina from solution, and that the action of a wide variety of substances can be explained in this manner.

The mineralogy of aluminous cement is complex, and the nature of the iron compounds present is still controversial. A large part of the ferrous iron is found in that portion of the clinker which solidifies last, forming a dark and usually opaque glass. X-ray evidence suggests that free ferrous oxide is present as wüstite in sub-microscopic form, thus accounting for the high refractive index of the glass. The unstable form of the compound $5 \mathrm{CaO} .3 \mathrm{Al}_{2} \mathrm{O}_{3}$ (probably more correctly formulated as $12 \mathrm{CaO} .7 \mathrm{Al}_{2} \mathrm{O}_{3}$ ) is somewhat remarkable in that it is the only member of the lime-alumina series showing pleochroism. It seems probable that, in aluminous cement at least, this is not a pure compound and that ferrous and other oxides are also present.

Many difficult problems still remain in the study of pozzolanas and of the physical structure of hydrated cements, but in connexion with the last of these there now appears to be a definite trend towards the view propounded by Le Chatelier that the hardening of Portland cement is primarily to be attributed to crystal formation.

During the period of the meeting and the tour which preceded it, visitors were entertained with much generosity by their Swedish hosts, and they owe to them a debt of gratitude, both for this and for their work in the organization of a very successful meeting.

\title{
Hydro-Kinetic Power Transmitter*
}

\author{
By Prof. F. C. Lea
}

\begin{abstract}
$\mathrm{T}$ HE problem of devising a hydro-kinetic power transmitter capable of performing effectively the functions both of a clutch and of a change speed gear has not infrequently been declared to be in. capable of solution. Tests of a transmitter evolved by Commendatore Piero Salerni indicate that the problem has now been satisfactorily solved. Within the range of variation of torque ratio requisite in an automobile or a heavy lorry, the transmitter in question performs the two functions effectively and has an advantage over the orthodox clutch and gear mechanism in efficiency, performance, simplicity, soundness of construction, and also cost. Furthermore, due to its inherent smoothness, it relieves the whole of the transmission system, from engine to road wheels, of shock loads, and thus reduces general maintenance.
\end{abstract}

A variable ratio hydro-kinetic transmitter consists essentially of three vaned elements, namely, a centrifugal pump or driving element, a turbine or driven element, and a reaction element. In the new transmitter the design of each of these elements involves important original conceptions, and of these conceptions that which relates to the centrifugal pump is of a fundamental nature and has important implications.

In a hydro-kinetic power transmitter which has to function under conditions varying as widely and unpredictably as in an automobile or a locomotive, the major causes of loss of energy have heretofore been : (1) unsteadiness of motion of the liquid medium within the ducts constituting the hydraulic circuit of the transmitter; (2) shock at the inlet of the driven element. Of these two causes of inefficiency, the first, although per se the lesser, is fundamental, since the second, though considerably graver, is in consequence of the first.

Since the liquid medium has to pass from a vaned driving element to a vaned driven element rotating relatively to one another at greatly differing and constantly varying speed, it is evident that, if losses

* Substance of a paper read before Section G (Engineering) of the British Association on August 22. of prohibitive magnitude are to be avoided, the rotational velocity with which the liquid emerges from the driving element must be so controlled that the direction in which the liquid impinges upon the receiving ends of the vanes of the driven element is maintained under all conditions, at an angle at which the liquid can be received without appreciable shock. Hitherto such control has not been achieved. Control of velocity variations implies, as a pre-requisite, steadiness of motion of the liquid, and it was held that in a centrifugal pump-and therefore in a hydro-kinetic power transmitter-the motion of the liquid could not be other than unsteady. Hence the accepted theory that under variable conditions a hydro-kinetic power transmitter can function with acceptable efficiency only within a comparatively narrow range of variation, and that efficient performance by a single transmitter of the widely differing functions of a clutch and of a variable ratio gear is an impossibility.

The fundamental characteristic of the new transmitter is that its driving element is a centrifugal pump of such a construction that the motion of the liquid within its ducts remains steady for the velocities that obtain in the ducts, with the result that a steady stream can be maintained within the hydraulie circuit of the transmitter under all conditions, and velocity variations can be, and are, adequately controlled. By virtue of this characteristic, both the fundamental and the consequential causes of loss of energy are virtually eliminated.

A series of laboratory tests have been carried out directed towards establishing the soundness of the hydraulic principle which governs the design and functioning of the machine, and I have had opportunities of observing and testing the performance of it in an automobile. The automobile tested was a standard $21 \cdot 6$ h.p. 1936 model fitted with the standard size tyres and the standard back axle ratio of $4 \cdot 1$ to 1 , the unladen weight of the vehicle being $29 \mathrm{cwt}$. The tests included trials under all driving conditions, that is, in traffic, in hilly country and on the open road. 
The hill-climbing capacity of the vehicle, and the rate of its acceleration at 'get-away', and under all other conditions, have proved to be appreciably greater than is advertised by the makers as achievable by the same model when driven with the standard clutch and gears; and, though acceleration was lively, it was always smooth and progressive, the torque ratio decreasing gradually as road speed increased. Inasmuch as with the transmitter there is neither de-clutching nor gear changing, optimum performance is achieved without involving gear changing skill on the driver's part. Passage from drive at the ratio of 1 to 1 to drive at higher ratios and vice versa is controlled solely by the accelerator, so that even at very low speed the car is driven at the ratio of 1 to 1 when acceleration is not needed, while torque increase takes place, whenever needed, the moment the accelerator pedal is pressed hard.

The hill-climbing performance of the vehicle was particularly impressive. A special hill-climbing trial was made on the 1 in 4 portion of the Brooklands test hill. The car was loaded with progressively greater weight until the final test, when a net load of 22 cwt. was put on the car. The car was then driven on to the 1 in 4 portion of the hill, was stopped and then restarted without difficulty on the same gradient and was driven comfortably to the top of the hill. This particular feature should prove of great advantage in heavy lorries, caterpillar tractors and the like.

I have carried out also a prolonged road test to ascertain the fuel consumption of a car fitted with the transmitter as compared with the fuel consumption of the same model when fitted with the standard clutch and gear box mechanism. This test has proved that with the transmitter a saving in fuel is effected.

As a result of tests in the laboratory and on the road, and of an examination of the working parts after they had been in use in an automobile over 65,000 miles, I have come to the conclusion that :

(1) The transmitter resolves in a satisfactory manner the problem of transmission of power at a torque ratio varying automatically and gradually within the whole range covered by the orthodox friction clutch and change speed gear mechanism.

(2) Its application in an automobile in substitution for the orthodox clutch and gear mechanism results in an improvement of the performance of the vehicle in get-away, general acceleration, hillclimbing, drive in traffic and drive on the open road.

(3) 'The mechanical construction of the transmitter is in every way simple and sound; as compared with the orthodox clutch and gear mechanism, it is simpler and less costly, and its overall efficiency (as measured by petrol consumption on a long run) is appreciably greater.

(4) Its scope transcends the field of the automobile and should include vehicles of every kind driven by internal combustion engines, both of the petrol and of the heavy oil type.

\section{Progress in Seismology}

$\mathrm{T}$ $\mathrm{HE}$ forty-third report of the Committee of Seismological Investigations of the British Association has just appeared, and shows great activity on the part of the members and others in numerous directions. The six Milne-Shaw seismographs belonging to the British Association have remained on loan to the seismological stations at Oxford (2), Edinburgh, Perth (Western Australia), and Cape Town (2). During the year, a Jagger shock recorder has been made for the Committee at Bristol under the supervision of Dr. C. F. Powell, and this instrument is to be set up at Dunira, near Comrie (the village in Perthshire which is famous for the prolonged series of minor earthquakes in the last century), thanks to the co-operation of Messrs. Macbeth and White. At Kew Observatory, the three Galitzin and two Wood-Anderson seismographs have been rehoused in a new underground building, and it is very satisfactory to learn that in their new position they are not affected by the wind as they were previously, due to the rocking of the building. A full description of the new housing is being published in a memoir written by Dr. A. W. Lee.

It is very satisfactory to learn that the work on British earthquakes which was carried on so long and so assiduously by Dr. C. Davison is now being continued by Dr. Dollar with the support of the Committee. Dr. Dollar is organizing the collection of data very thoroughly, and already has collected information from numerous collaborators concerning six earthquakes, four subsidences and mine-shakes, two explosions and six earth tremors felt by people in Great Britain since July 1, 1937. The earthquakes had the following dates and epicentres: 1937, July 9 at Walsall in Staffordshire ; 1937 , July 20 , in Perthshire; 1937, September 8, at Horsham in Sussex ; 1937, December 4, at Comrie in Perthshire; 1938, March 21, in S.E. Edinburgh, and 1938, June 11, with epicentre at Ghent in Belgium. The latter was by far the most important, though the only damage done in Great Britain appears to have been a single fall of a few tiles at Herne Bay, in Kent. The low rumbling earth-sound was imperceptible west of London. In the West Indies, earthquakes for the time being appear to be less frequent. The Weichert seismograph and eight Jagger shock recorders are still in operation in the care of $\mathrm{Mr}$. Kelsick, who is making regular reports. From August until November 1937 about forty earthquakes were reported by observers in Dominica, and valuable reports on the geological structure of the island and on the distribution of earthquake centres were written by Mr. A. G. Macgregor and Dr. C. F. Powell.

The work of the International Seismological Summary is being actively pursued at Oxford by Mr. J. S. Hughes and Miss E. F. Bellamy. The I.S.S. has now been prepared in manuscript as far as July 1933, and January, February and March are in the press. From January 1933 onwards an attempt is being made to distinguish between anaseisms and kataseisms. Theoretical work on southern earthquakes and the core waves, periodicities, and deepfocus shocks has been done by the indefatigable Dr. H. Jeffreys, and on the travel times of $P$ and $S$ waves by Dr. A. W. Lee. Dr. E. C. Bullard is likely to undertake work on the thickness of the strata overlying the continental shelf round the British Isles. 